\title{
On the Solutions of the Higher Order Fractional Differential Equations of Riesz Space Derivative with Anti-Periodic Boundary Conditions
}

\author{
Şuayip Toprakseven ${ }^{1 *}$
}

\begin{abstract}
We present existence and uniqueness results for a class of higher order anti-periodic fractional boundary value problems with Riesz space derivative which is two-sided fractional operator. The obtained results are established by applying some fixed point theorems. Various numerical examples are given to illustrate the obtained results.

Keywords: Anti periodic boundary conditions, existence, fixed point Theorem, fractional boundary value problem Riesz Caputo derivative

2010 AMS: 34A08, 34A40, Secondary: 26D10, 34C10, 33E12
\end{abstract}

${ }^{1}$ Faculty of Engineering, Department of Computer Science, Artvin Çoruh University, Artvin, 08100, Turkey, ORCID:0000-0003-3901-9641

*Corresponding author: topraksp@artvin.edu.tr

Received: 30 October 2021, Accepted: 16 December 2021, Available online: xxxx

\section{Introduction}

Recently, many researchers have investigated a large range of problems including fractional differential equations. A variety of scientific areas such as physics, polymer rheology, regular variation in thermodynamics, biophysics, blood flow phenomena, aerodynamics, electro-dynamics of complex medium, viscoelasticity, biology, control theory, etc involve fractional differential equations. Some applications and detailed explanation of fractional differential equations can be found in the books [1, 2, 3] and references $[7,29,16]$. Geometric and physical interpretation of fractional differentiation and integration can be found in the paper [27]. Existence results for fractional differential equations have studied and developed by many authors; see the books $[26,4,2]$ and references $[11,12,24,15,9,26,4,2,17,18,19,30,31,39,41,42,43,44,45]$ and references therein.

Much of recent works on fractional boundary/initial value problems involve Riemann-Lioville and Caputo derivatives in the literature. Unfortunately, these fractional operators are one-sided operators which hold either past or future memory effects. Unlike these fractional operators, the Riesz space fractional operator is two-sided operator which holds both the history and future non-local memory effects. This is important in the mathematical modelling for physical processes on a finite domain because the present states depend both on the past and future memory effects. As an example, Riesz fractional derivative has been used for the memory effects in both past and future concentrations in the anomalous diffusion problem [13,5].

Numerical solutions of the fractional calculus, specifically in the anomalous diffusion that involves the Riesz derivative have been presented in [13, 8, 5, 38]. Analytical and numerical solutions for fractional differential equations using different definitions for fractional derivatives and integrals have been proposed and studied in the literature [28, 32, 33, 34, 35, 21, 36, 37]. Recently, there are papers on existence and positive solutions for the fractional boundary value problems with the Riesz-Caputo derivative [14, 25, 20].

The mathematical modelling of many physical phenomena can be expressed in terms of anti-periodic boundary value 
problems [10]. Recently, a large amount of papers are devoted to anti-periodic boundary value problems, for example, see $[22,23]$ and references therein.

In this paper, we study the existence and uniqueness of solutions for the following anti-periodic boundary value problem of the Riesz-Caputo fractional differential equations

$$
\begin{aligned}
{ }_{0}^{R C} D_{T}^{v} u(\eta) & =F(\eta, u(\eta)) \quad v \in(2,3], \quad 0 \leq \eta \leq T, \\
u(0)+u(T) & =0, \quad u^{\prime}(0)+u^{\prime}(T)=0, \quad u^{\prime \prime}(0)+u^{\prime \prime}(T)=0,
\end{aligned}
$$

where ${ }_{0}^{R C} D_{T}^{v}$ is the Riesz-Caputo derivative defined below and $F:[0, T] \times \mathbb{R} \rightarrow \mathbb{R}$ is a continuous function.

The remainder of paper is organized as follows. Section 2 introduces some preliminaries, definitions and lemmas which are useful in proving main results. Section 3 provides some sufficient conditions for the existence and the uniqueness of solutions of the problem (1.1) with anti-periodic boundary conditions. Finally, some numerical examples are given to illustrate the applications of the main results in the last section.

\section{Preliminaries}

This section is devoted to some important definitions and lemmas that will be needed in the sequel.

Definition 2.1. [26] Let $v>0$. The left and right Riemann-Liouville fractional integral of a function $f \in C[0, T]$ of order $v$ defined as, respectively

$$
\begin{aligned}
& I_{0}^{v} f(x)=\frac{1}{\Gamma(v)} \int_{0}^{x}(x-s)^{v-1} f(s) d s, \quad x \in[0, T] . \\
& { }_{T} I^{v} f(x)=\frac{1}{\Gamma(v)} \int_{x}^{T}(s-x)^{v-1} f(s) d s, \quad x \in[0, T] .
\end{aligned}
$$

Definition 2.2. (Riesz Fractional Integral) Let $v>0$. The Riesz fractional integral of a function $f \in C[0, T]$ of order $v$ defined as

$$
{ }_{0} I_{T}^{v} f(x)=\frac{1}{2 \Gamma(v)} \int_{0}^{T}|x-s|^{v-1} f(s) d s, \quad x \in[0, T]
$$

Note that the Riesz fractional integral operator can be written as

$$
{ }_{0} I_{T}^{v} f(x)=\frac{1}{2}\left(I_{0}^{v} f(x)+{ }_{T} I^{v} f(x)\right)
$$

Definition 2.3. [26] Let $v \in(n, n+1], n \in \mathbb{N}$. The left and right Caputo fractional derivative of a function $f \in C^{n+1}[0, T]$ of order $v$ defined as, respectively

$$
\begin{aligned}
& { }_{0}^{C} D_{x}^{v} f(x)=\frac{1}{\Gamma(n+1-v)} \int_{0}^{x}(x-s)^{n-v} f^{(n+1)} d s=\left(I_{0}^{n+1-v} D^{n+1}\right) u(x) . \\
& { }_{x}^{C} D_{T}^{v} f(x)=\frac{(-1)^{n+1}}{\Gamma(n+1-v)} \int_{x}^{T}(s-x)^{n-v} f^{(n+1)} d s=(-1)^{n+1}\left({ }_{T} I^{n+1-v} D^{n+1}\right) u(x) .
\end{aligned}
$$

where $D$ is the ordinary differential operator.

Definition 2.4. Let $v \in(n, n+1], n \in \mathbb{N}$. The Riesz-Caputo fractional derivative ${ }_{0}^{R C} D^{v} f$ of order $v$ of a function $f \in C^{n+1}[0, T]$ defined by

$$
\begin{aligned}
{ }_{0}^{R C} D_{T}^{v} f(x) & =\frac{1}{\Gamma(n+1-v)} \int_{0}^{T}|x-s|^{n-v} f^{(n+1)}(s) d s \\
& =\frac{1}{2}\left({ }_{0}^{C} D_{x}^{v} f(x)+(-1)^{n+1 C} D_{T}^{v} f(x)\right) \\
& =\frac{1}{2}\left(\left(I_{0}^{n+1-v} D^{n+1}\right) u(x)+(-1)^{n+1}\left({ }_{T} I^{n+1-v} D^{n+1}\right) u(x)\right)
\end{aligned}
$$


Lemma 2.5. [26] Let $f \in C^{n}[0, T]$ and $v \in(n, n+1]$. Then we have the following relations

$$
\begin{aligned}
& I_{0}^{v C} D_{x}^{v} f(x)=f(x)-\sum_{k=0}^{n-1} \frac{f^{(k)}(a)}{k !}(x-a)^{k}, \\
& { }_{T} I_{x}^{V C} D_{T}^{v} f(x)=f(x)-\sum_{k=0}^{n-1} \frac{(-1)^{k} f^{(k)}(b)}{k !}(b-x)^{k} .
\end{aligned}
$$

In the case when $v \in(2,3]$ and $f(x) \in C^{3}(0, T)$ we have

$$
\begin{aligned}
{ }_{0} I_{T}^{v}{ }_{0} D_{T}^{v} f(x)= & \frac{1}{2}\left(I_{00}^{v C} D_{x}^{v} f(x)-{ }_{T} I_{x}^{v C} D_{T}^{v} f(x)\right) \\
= & f(x)-\frac{1}{2}(f(0)+f(T))-\frac{1}{2}\left(f^{\prime}(0)+f^{\prime}(T)\right) x+\frac{T}{2} f^{\prime}(T) \\
& -\frac{1}{4}\left(f^{\prime \prime}(0)+f^{\prime \prime}(T)\right) x^{2}-\frac{T^{2}-2 T x}{4} f^{\prime \prime}(T) .
\end{aligned}
$$

The following fixed point theorems will be needed to establish the existence results.

Theorem 2.6. [6] Let $M$ be a closed convex and nonempty subset of a Banach space X. Let A,B be the operators such that

(i) $A x+B y \in M$ whenever $x, y \in M$;

(ii) A is compact and continuous;

(iii) $B$ is a contraction mapping.

Then there exists $u \in M$ such that $u=A u+B u$.

Theorem 2.7. [6] Let $X$ be a Banach space. Assume that $O$ is an open bounded subset of $X$ with $\theta \in O$ and let $T: \bar{O} \rightarrow X$ be a completely continuous operator such that

$$
\|T u\| \leq\|u\|, \quad \forall u \in \partial O .
$$

Then $T$ has a fixed point in $\bar{O}$.

Lemma 2.8. Assume that $g \in C([0, T], \mathbb{R})$. A unique solution $u \in C^{3}([0, T])$ of the following fractional boundary problem

$$
\begin{aligned}
{ }_{0}^{R C} D_{T}^{v} u(\eta) & =g(\eta) \quad v \in(2,3], \quad 0 \leq \eta \leq T, \\
u(0)+u(T) & =0, \quad u^{\prime}(0)+u^{\prime}(T)=0, \quad u^{\prime \prime}(0)+u^{\prime \prime}(T)=0,
\end{aligned}
$$

is given as

$$
\begin{aligned}
u(\eta)= & \frac{2 T^{2}-2 T \eta}{4 \Gamma(v-2)} \int_{0}^{T}(T-s)^{v-3} g(s) d s-\frac{T}{2 \Gamma(v-1)} \int_{0}^{T}(T-s)^{v-2} g(s) d s \\
& +\frac{1}{\Gamma(v)} \int_{0}^{T}|\eta-s|^{v-1} g(s) d s .
\end{aligned}
$$

Proof. We infer from (2.2) and (2.3) that

$$
\begin{aligned}
u(\eta)= & \frac{1}{2}(u(0)+u(T))+\frac{1}{2}\left(u^{\prime}(0)+u^{\prime}(T)\right) \eta-\frac{T}{2} u^{\prime}(T) \\
& \frac{1}{4}\left(u^{\prime \prime}(0)-u^{\prime \prime}(T)\right) \eta^{2}+\frac{T^{2}-2 T \eta}{4} u^{\prime \prime}(T)+\frac{1}{\Gamma(v)} \int_{0}^{T}|\eta-s|^{v-1} g(s) d s .
\end{aligned}
$$

The anti-periodic boundary conditions $u(0)+u(T)=0, \quad u^{\prime}(0)+u^{\prime}(T)=0, \quad u^{\prime \prime}(0)+u^{\prime \prime}(T)=0$ imply that

$$
u(\eta)=-\frac{T}{2} u^{\prime}(T)+\frac{T^{2}-2 T \eta}{4} u^{\prime \prime}(T)+\frac{1}{\Gamma(v)} \int_{0}^{T}|\eta-s|^{v-1} g(s) d s .
$$


Then,

$$
\begin{aligned}
& u^{\prime}(\eta)=-\frac{T}{2} u^{\prime \prime}(T)+\frac{1}{\Gamma(v-1)} \int_{0}^{\eta}(\eta-s)^{v-2} g(s) d s-\frac{1}{\Gamma(v-1)} \int_{\eta}^{T}(s-\eta)^{v-2} g(s) d s \\
& u^{\prime \prime}(\eta)=\frac{1}{\Gamma(v-2)} \int_{0}^{\eta}(\eta-s)^{v-3} g(s) d s+\frac{1}{\Gamma(v-2)} \int_{\eta}^{T}(s-\eta)^{v-3} g(s) d s .
\end{aligned}
$$

Hence, we have

$$
\begin{aligned}
u^{\prime}(T) & =-\frac{T}{2}\left(\frac{1}{\Gamma(v-2)} \int_{0}^{T}(T-s)^{v-3} g(s) d s\right)+\frac{1}{\Gamma(v-1)} \int_{0}^{T}(T-s)^{v-2} g(s) d s, \\
u^{\prime \prime}(T) & =\frac{1}{\Gamma(v-2)} \int_{0}^{T}(T-s)^{v-3} g(s) d s .
\end{aligned}
$$

Plugging the equations in (2.7) into (2.6) gives

$$
u(\eta)=\frac{2 T^{2}-2 T \eta}{4 \Gamma(v-2)} \int_{0}^{T}(T-s)^{v-3} g(s) d s-\frac{T}{2 \Gamma(v-1)} \int_{0}^{T}(T-s)^{v-2} g(s) d s \quad+\frac{1}{\Gamma(v)} \int_{0}^{T}|\eta-s|^{v-1} g(s) d s
$$

which completes the proof.

\section{Existence of Solutions}

We prove the main results of the paper in this section. Let $C[0, T]$ be the space of continuous functions $u$ defined on $[0, T]$ with the norm $\|u\|=\sup _{\eta \in[0, T]}|u(\eta)|$. We assume the following conditions on $F$ are satisfied.

(H1) $F$ satisfies a Lipschitz condition in the second variable, that is,

$$
|F(\eta, u)-F(\eta, v)| \leq L|u-v|, \forall \eta \in[0, T], u, v \in \mathbb{R}
$$

(H2) $F$ is dominated by a $L^{1}$ function, that is,

$$
|F(\eta, u)| \leq \ell(\eta), \forall(\eta, u) \in[0, T] \times \mathbb{R}, \text { and } \ell \in L^{1}\left([0, T], \mathbb{R}^{+}\right)
$$

Theorem 3.1. Let $F \in C([0, T] \times \mathbb{R}, \mathbb{R})$ satisfy the assumption $(H 1)$ with

$$
L \leq \frac{2 \Gamma(v+1)}{T^{v}(8+v(v+1))}
$$

Then the problem (1.1) has a unique solution.

Proof. We convert the problem (1.1) into a fixed point solution of operator $\mathscr{T}: C([0, T], \mathbb{R}) \rightarrow C([0, T], \mathbb{R})$ defined by

$$
\begin{aligned}
(\mathscr{T} u)(\eta)= & \frac{2 T^{2}-2 T \eta}{4 \Gamma(v-2)} \int_{0}^{T}(T-s)^{v-3} F(s, u(s)) d s-\frac{T}{2 \Gamma(v-1)} \int_{0}^{T}(T-s)^{v-2} F(s, u(s)) d s \\
& +\frac{1}{\Gamma(v)} \int_{0}^{T}|\eta-s|^{v-1} F(s, u(s)) d s, \quad \eta \in[0, T] .
\end{aligned}
$$

We shall prove that the operator $\mathscr{T}$ has a fixed point by showing that $\mathscr{T}$ is a contraction. To this end, we first demonstrate that $\mathscr{T} S_{r} \subset S_{r}$ where $S_{r}=\{u \in C([0, T], \mathbb{R}):\|u\| \leq r\}$ with $r \geq \frac{K T^{v}(8+v(v+1))}{2 \Gamma(v+1)}$ and $K:=\sup _{\eta \in[0, T]}|F(\eta, 0)|$. For $u \in S_{r}$, we 
have

$$
\begin{aligned}
|(\mathscr{T} u)(\eta)| \leq & \frac{2 T^{2}-2 T \eta}{4 \Gamma(v-2)} \int_{0}^{T}(T-s)^{v-3}|F(s, u(s))| d s+\frac{T}{2 \Gamma(v-1)} \int_{0}^{T}(T-s)^{v-2}|F(s, u(s))| d s \\
& +\frac{1}{\Gamma(v)} \int_{0}^{T}|\eta-s|^{v-1}|F(s, u(s))| d s \\
\leq & \frac{2 T^{2}-2 T \eta}{4 \Gamma(v-2)} \int_{0}^{T}(T-s)^{v-3}(|F(s, u(s))-F(s, 0)|+|F(s, 0)|) d s+\frac{T}{2 \Gamma(v-1)} \int_{0}^{T}(T-s)^{v-2}(\mid F(s, u(s)) \\
& -F(s, 0)|+| F(s, 0) \mid) d s+\frac{1}{\Gamma(v)} \int_{0}^{T}|\eta-s|^{v-1}(|F(s, u(s))-F(s, 0)|+|F(s, 0)|) d s \\
\leq & (L r+K)\left(\frac{2 T^{2}-2 T \eta}{4 \Gamma(v-2)} \int_{0}^{T}(T-s)^{v-3} d s+\frac{T}{2 \Gamma(v-1)} \int_{0}^{T}(T-s)^{v-2} d s+\frac{1}{\Gamma(v)} \int_{0}^{T}|\eta-s|^{v-1} d s\right) \\
\leq & (L r+K)\left(\frac{T^{v}}{\Gamma(v+1)}\left(2+\frac{v(v+1)}{4}\right)\right) \leq r .
\end{aligned}
$$

Next, for $u, v \in C([0, T), \mathbb{R})$ and for any $\eta \in[0, T]$, we get

$$
\begin{aligned}
& |(\mathscr{T} u)(\eta)-(\mathscr{T} v)(\eta)| \\
\leq & \frac{2 T^{2}-2 T \eta}{4 \Gamma(v-2)} \int_{0}^{T}(T-s)^{v-3}|F(s, u(s))-F(s, v(s))| d s \\
& +\frac{T}{2 \Gamma(v-1)} \int_{0}^{T}(T-s)^{v-2}|F(s, u(s))-F(s, v(s))| d s+\frac{1}{\Gamma(v)} \int_{0}^{T}|\eta-s|^{v-1}|F(s, u(s))-F(s, v(s))| d s \\
\leq & L\|u-v\|\left(\frac{2 T^{2}-2 T \eta}{4 \Gamma(v-2)} \int_{0}^{T}(T-s)^{v-3} d s+\frac{T}{2 \Gamma(v-1)} \int_{0}^{T}(T-s)^{v-2} d s+\frac{1}{\Gamma(v)} \int_{0}^{T}|\eta-s|^{v-1} d s\right) \\
& \leq\left(\frac{L T^{v}}{\Gamma(v+1)}\left(2+\frac{v(v+1)}{4}\right)\right)\|u-v\|<\|u-v\| .
\end{aligned}
$$

This shows that $\mathscr{T}$ is a contraction. Therefore, the Banach fixed point theorem tells us $\mathscr{T}$ has a fixed point which is a solution to the problem (1.1).

Theorem 3.2. Let $F \in C([0, T] \times \mathbb{R}, \mathbb{R})$ be a completely continuous function. Assume that the conditions (H1) and (H2) hold with $\frac{L T^{v}(v+1)}{4 \Gamma(v)}<1$. Then the fractional boundary problem with anti-periodic boundary conditions (1.1) has a solution on $[0, T]$.

Proof. Let $S_{r}=\left\{u \in C([0, T], \mathbb{R}):\|u\| \leq r\right.$ be the ball of radius $r$ with $r \geq \frac{\|\ell\|_{L^{1}} T^{v}}{\Gamma(v+1)}\left(2+\frac{v(v+1)}{4}\right)$, where $\|\ell\|_{L^{1}}=\int_{0}^{T}|\ell(s)| d s$. We define two operator $\mathscr{F}$ and $\mathscr{S}$ on $S_{r}$ given by

$$
\begin{aligned}
& (\mathscr{F} u)(\eta):=\frac{1}{\Gamma(v)} \int_{0}^{T}|\eta-s|^{v-1} F(s, u(s)) d s \\
& (\mathscr{S} u)(\eta):=\frac{2 T^{2}-2 T \eta}{4 \Gamma(v-2)} \int_{0}^{T}(T-s)^{v-3} F(s, u(s)) d s-\frac{T}{2 \Gamma(v-1)} \int_{0}^{T}(T-s)^{v-2} F(s, u(s)) d s .
\end{aligned}
$$

For any $u, v \in S_{r}$, as above, we have

$$
\|\mathscr{F} u+\mathscr{S} v\| \leq \frac{\|\ell\|_{L^{1}} T^{v}}{\Gamma(v+1)}\left(2+\frac{v(v+1)}{4}\right) \leq r
$$

Hence, it follows that $\mathscr{F} u+\mathscr{S} v \in S_{r}$ whenever $u, v \in S_{r}$. It can easily be shown that $\mathscr{S}$ is a contraction using the assumption 
$\frac{L T^{v}(v+1)}{4 \Gamma(v)}<1$. The continuity of $\mathscr{F}$ follows from the continuity of $F$. Moreover, $\mathscr{F}$ is uniformly bounded on $S_{r}$ as follows.

$$
\begin{aligned}
|(\mathscr{F} u)(\eta)| & \leq \frac{1}{\Gamma(v)} \int_{0}^{\eta}(\eta-s)^{v-1}|F(s, u(s))| d s+\frac{1}{\Gamma(v)} \int_{\eta}^{T}(s-\eta)^{v-1}|F(s, u(s))| d s \\
& \leq \frac{\|\ell\|_{L_{1}}}{\Gamma(v)}\left(\int_{0}^{\eta}(\eta-s)^{v-1} d s+\int_{\eta}^{T}(s-\eta)^{v-1} d s\right) \\
& \leq \frac{\|\ell\|_{L_{1}}}{\Gamma(v+1)}\left(\eta^{v}+(T-\eta)^{v}\right) \leq \frac{2\|\ell\|_{L_{1}} T^{v}}{\Gamma(v+1)}
\end{aligned}
$$

We now show that the operator $\mathscr{F}$ is compact on $S_{r}$. For $u \in S_{r}$, we first estimate the derivative $(\mathscr{F} u)^{\prime}(\eta)$ :

$$
\begin{aligned}
\left|(\mathscr{F} u)^{\prime}(\eta)\right| & \leq \frac{1}{\Gamma(v-1)} \int_{0}^{\eta}(\eta-s)^{(v-2)}|F(s, u(s))| d s+\frac{1}{\Gamma(v-1)} \int_{\eta}^{T}(s-\eta)^{(v-2)}|F(s, u(s))| d s \\
& \leq\left(\frac{\eta^{v-1}}{\Gamma(v)}+\frac{(T-\eta)^{v-1}}{\Gamma(v)}\right) L \leq \frac{2 T^{v-1} L}{\Gamma(v)}:=\beta_{T, L, v}
\end{aligned}
$$

where $\beta_{T, L, v}$ is independent of the function $u$. Therefore, for any $\eta_{1}, \eta_{2} \in[0, T]$ with $\eta_{1}<\eta_{2}$, we have

$$
\left|(\mathscr{F} u)\left(\eta_{1}\right)-(\mathscr{F} u)\left(\eta_{2}\right)\right|=\int_{\eta_{1}}^{\eta_{2}}\left|(\mathscr{F} u)^{\prime}(s)\right| d s \leq \beta_{T, L, v}\left(\eta_{2}-\eta_{1}\right) .
$$

Hence, $\mathscr{F}$ is relatively compact on $S_{r}$. It follows form Arzela Ascoli Theorem that $\mathscr{F}$ is compact on $S_{r}$. As a consequence of Theorem 2.6, we infer that $\mathscr{F}+\mathscr{S}$ has a fixed point which is a solution of the problem $(1.1)$ on $[0, T]$. Thus the proof is completed.

Theorem 3.3. Assume that $\lim _{u \rightarrow 0} \frac{F(\eta, u)}{u}=0$. Then the problem (1.1) has one solution.

Proof. $\lim _{u \rightarrow 0} \frac{F(\eta, u)}{u}=0$ implies that there is a $\delta>0$ such that $|F(\eta, u)| \leq \varepsilon|u|$ for $0<|u|<\delta$, where $\varepsilon$ is chosen such that

$$
\left(\frac{T^{v}}{\Gamma(v+1)}\left(2+\frac{v(v+1)}{4}\right)\right) \varepsilon \leq 1 .
$$

Set $S_{r}=\left\{u \in C([0, T], \mathbb{R}):\|u\|<r\right.$ and let $u \in \partial S_{r}$, that is $\|u\|=r$. As before, the continuity of the operator $\mathscr{T}$ follows from the continuity of $F$, and, as before, it can be shown that $\mathscr{T}=\mathscr{F}+\mathscr{S}$ is bounded on $S_{r}$. Note that $\left|(\mathscr{T} u)^{\prime}=(\mathscr{F} u)^{\prime}+(\mathscr{S} u)^{\prime}\right|$ where $(\mathscr{F} u)^{\prime}$ is given by $(3.1)$ and $(\mathscr{S} u)^{\prime}$ is given as

$$
\left|(\mathscr{S} u)^{\prime}(\eta)\right|=\frac{T}{2 \Gamma(v-2)} \int_{0}^{T}(T-s)^{(v-3)}|F(s, u(s))| d s \leq \frac{T^{v-1} L}{\Gamma(v-1)} .
$$

Hence,

$$
\left|(\mathscr{T} u)^{\prime}(\eta)\right| \leq \frac{(v+1) T^{v-1} L}{\Gamma(v-1)}:=L_{1} .
$$

Therefore, for $\eta_{1}, \eta_{2} \in[0, T]$ with $\eta_{1}<\eta_{2}$, we have

$$
\left|(\mathscr{T} u)^{\prime}\left(\eta_{1}\right)-(\mathscr{T} u)^{\prime}\left(\eta_{2}\right)\right| \leq \int_{\eta_{1}}^{\eta_{2}}\left|(\mathscr{T} u)^{\prime}(s)\right| d s \leq L_{1}\left(\eta_{2}-\eta_{1}\right) .
$$

We deduce that $\mathscr{T}$ is equicontinuous on $[0, T]$. Hence, in view of the Arzela-Ascoli theorem, the operator $\mathscr{T}$ is completely continuous. Morevover, we have

$$
|(\mathscr{T} u)(\eta)| \leq\left(\frac{T^{v}}{\Gamma(v+1)}\left(2+\frac{v(v+1)}{4}\right)\right) \varepsilon\|u\|,
$$

which implies $\|\mathscr{T} u\| \leq\|u\|$ for $u \in \partial S_{r}$ in light of (3.2). As a consequence of Theorem 2.7, the operator $\mathscr{T}$ has a fixed point which is solution of the problem (1.1). 
Remark 3.4. The results in this paper can be applied to obtain the existence results for nonlinear third-order ordinary differential equations with anti-periodic boundary conditions [40] by taking $v=3$

$$
\begin{aligned}
& u^{\prime \prime \prime \prime}(\eta)=F(\eta, u(\eta)) \quad 0 \leq \eta \leq T, \\
& u(0)+u(T)=0, \quad u^{\prime}(0)+u^{\prime}(T)=0, \quad u^{\prime \prime}(0)+u^{\prime \prime}(T)=0 .
\end{aligned}
$$

\section{Numerical Examples}

In this section, numerical examples are given to show the applications of the result of this paper.

Example 4.1. Consider the following fractional boundary problem with anti-periodic boundary conditions

$$
\begin{aligned}
{ }_{0}^{R C} D_{1}^{\frac{5}{2}} u(\eta) & =\frac{1}{(2+\eta)^{2}} \frac{u(\eta)}{2+u(\eta)} \quad 0 \leq \eta \leq 1, \\
u(0)+u(1) & =0, \quad u^{\prime}(0)+u^{\prime}(1)=0, \quad u^{\prime \prime}(0)+u^{\prime \prime}(1)=0 .
\end{aligned}
$$

Here, $F(s, u(s))=\frac{1}{(2+\eta)^{2}} \frac{u(\eta)}{2+u(\eta)}, \quad T=1$ and $v=\frac{5}{2}$. We have $|F(s, u)-F(s, v)| \leq \frac{1}{4}\|u-v\|$,hence the condition (H1) is fulfilled with $L=\frac{1}{4}$. Also, we calculate $\frac{L T^{v}}{\Gamma(v+1)}\left(2+\frac{v(v+1)}{4}\right) \approx 0.3150<1$. Therefore, the fractional boundary value problem (4.1) has a solution by Theorem 3.1.

Example 4.2. Consider the following fractional boundary problem with anti-periodic boundary conditions

$$
\begin{aligned}
{ }_{0}^{R C} D_{1}^{v} u(\eta) & =u^{3 / 2}(\eta)+3(\eta+2)(u(\eta)-\tan u(\eta)), \quad v \in(2,3], \quad 0 \leq \eta \leq 1, \\
u(0)+u(1) & =0, \quad u^{\prime}(0)+u^{\prime}(1)=0, \quad u^{\prime \prime}(0)+u^{\prime \prime}(1)=0,
\end{aligned}
$$

where $\left.F(s, u(s))=u^{3 / 2}(\eta)\right)+3(\eta+2)(u(\eta)-\tan u(\eta)), \quad T=1$ and $v \in(2,3]$ is any real number. We have $\lim _{u \rightarrow 0} \frac{F(\eta, u)}{u}=$ 0 , hence the condition of Theorem 3.3 holds. As a result of Theorem 3.3, the fractional boundary value problem (4.2) has at least one solution.

\section{Conclusion}

This paper concerns with the existence and uniqueness for fractional differential equations with the Riesz space with antiperiodic boundary conditions in Banach spaces. With the help of Banach's contraction principle and some fixed point theorems, existence results have been presented. As a special value of the fractional order, the results are extended to nonlinear third order ordinary differential equation with anti-periodic boundary conditions. Some examples are given to illustrate the theoretical results.

\section{Acknowledgement}

The authors would like to express their sincere thanks to the editor and the anonymous reviewers for their helpful comments and suggestions.

\section{Funding}

There is no funding for this work.

\section{Availability of data and materials}

Not applicable.

\section{Competing interests}

The authors declare that they have no competing interests. 


\section{Author's contributions}

All authors contributed equally to the writing of this paper. All authors read and approved the final manuscript.

\section{References}

[1] R. L. Magin, Fractional calculus in bioengineering, Begell House Publisher, Inc., Connecticut, 2006.

[2] I. Podlubny, Fractional Differential Equations, Academic Press, San Diego, CA, 1999.

[3] G. M. Zaslavsky, Hamiltonian chaos and fractional dynamics, Oxford University Press, Oxford 2005.

[4] K. Miller, B. Ross, An introduction to the fractional calculus and fractional differential equations, John Wiley, NY, 1993.

[5] S. Shen, F. Liu, V. Anh, Numerical approximations and solution techniques for the Caputo-time Riesz-Caputo fractional advection-diffusion equation, Numer. Algorithms, 56 (2011), 383-403.

[6] D. R. Smart, Fixed point Theorems, Cambridge University Press, Cambridge 1980.

[7] C. Pinto, A. R. M. Carvalho, New findings on the dynamics of HIV and TB coinfection models, Appl. Math. Comput., 242(2014), 36-46.

[8] R. Gorenflo, F. Mainardi, D. Moretti, G. Pagnini, P. Paradisi, Discrete random walk models for space-time fractional diffusion, Chem. Phys., 284 (2012), 521-541.

${ }^{[9]}$ L. Guo, L. Liu, W. Ye, Uniqueness of iterative positive solutions for the singular fractional differential equations with integral boundary conditions, Comput. Math. Appl., 59(8) (2010), 2601-2609.

[10] J. W. Negele, E. Vogt (Eds.), Volume 23 of advances in the physics of particles and nuclei, Advances in nuclear physics, Springer Science and Business Media, 1996.

[11] R. Agarwal, D, O’Regan, S. Stanek, Positive solutions for Dirichlet problems of singular nonlinear fractional differential equations, J. Math. Anal. Appl., 371 (2010), 57-68.

[12] A. Babakhani, V. Gejji, Existence of positive solutions of nonlinear fractional differential equations, J. Math. Anal.Appl., 278 (2003), 434-442.

[13] C. Celik, M. Duman, Crank-Nicolson method for the fractional diffusion equation with the Riesz fractional derivative, J. Comput. Phys., 231 (2012), 1743-1750.

[14] F. Chen, A. Chen, X. Wu, Anti-periodic boundary value problems with Riesz-Caputo derivative, Adv. Dif. Eq., 2019 (2019), 119.

[15] M.Darwish, S. Ntouyas, On initial and boundary value problems for fractional order mixed type functional differential inclusion, Comput. Math. Appl., 59 (2010), 1253-1265.

[16] H. Sun, S. Hu, Y. Chen, W. Chen, Z. Yu, A dynamic-order fractional dynamic system Chinese Phys. Lett., 30 (2013), Article 046601 pp.4.

[17] S.Toprakseven, Existence and uniqueness of solutions to anti-periodic Riezs-Caputo impulsive fractional boundary value problems, Tbil. Math. J. 14(1) (2021), 71-82.

[18] S. Toprakseven, Existence and uniqueness of solutions to Riesz-Caputo impulsive fractional boundary value problems, Journal of Interdisciplinary Mathematics, (2021), DOI: 10.1080/09720502.2020.1826629.

[19] S. Toprakseven, Positive solutions for two-point conformable fractional differential equations by monotone iterative scheme, TWMS J. App. Eng. Math., 11(1) (2021), 289-301.

[20] S. Toprakseven, Solvability of fractional boundary value problems for a combined caputo derivative, Konuralp J. Math., 9(1) (2021), 119-126.

[21] F. Usta, M. Z. Sarıkaya, The analytical solution of Van der Pol and Lienard differential equations within conformable fractional operator by retarded integral inequalities, Demo. Math., 52(1) (2019), 204-212.

${ }^{[22]}$ B. Ahmad, Existence of solutions for fractional differential equations of order $q \in(2,3]$ with anti-periodic boundary conditions, J. Appl. Math. Comput., 34 (2010), 385-391.

[23] Y. Chen, J.J. Nieto, D. O'Regan, Anti-periodic solutions for evolution equations associated with maximal monotone mappings, Appl. Math. Lett., 24 (3) (2011), 302-307.

[24] Y. Cui, Uniqueness of solution for boundary value problems for fractional differential equations, Appl. Math. Lett., 51 (2016), 48-54. 
[25] C. Gu, G. Wu, Positive solutions of fractional differential equations with the Riesz space derivative, Appl. Math. Lett., 95 (2019), 59-64.

[26] A. Kilbas, H. H. Srivastava, J. Trujillo, Theory and applications of fractional differential equations, vol. 204, North-Holland mathematics studies, Elsevier, Amsterdam, 2006.

[27] I. Podlubny, Geometric and physical interpretation of fractional integration and fractional differentiation, Fract. Calculus Appl. Anal., 5 (2002), 367-386.

[28] M. Z. Sarıkaya, F. Usta, On comparison theorems for conformable fractional differential equations, Int. J. Anal. App., 12(2) (2016), 207-214.

[29] Sierociuk, T. Skovranek, M. Macias, I. Podlubny, I. Petras, A. Dzielinski, P. Ziubinski, Diffusion process modeling by using fractional-order models, Appl. Math. Comput., 15 (257) (2015), 2-11.

[30] S. Toprakseven, The existence and uniqueness of initial-boundary value problems of the fractional Caputo-Fabrizio differential equations, Uni. J. Math. App., 2 (2) (2019), 100-106.

[31] S. Toprakseven, The existence of positive solutions and a Lyapunov-type inequality for boundary value problems of the fractioanl Caputo-Fabrizio differential equations, Sigma J. Eng. Nat. Sci., 37 (4) (2019), 1125-1133.

[32] F. Usta, Numerical analysis of fractional Volterra integral equations via Bernstein approximation method, J. Comput. Appl. Math., 384(2021), 113198, DOI: 10.1016/j.cam.2020.113198.

[33] F. Usta, Fractional type Poisson equations by radial basis functions Kansa approach, J. Ineq. Special Func., 7(4) (2016), 143-149.

[34] F. Usta, Numerical solution of fractional elliptic PDE's by the collocation method, Applications and Applied Mathematics: An International Journal, 12(1) (2017), 470- 478.

[35] F. Usta, H. Budak, M. Z. Sarıkaya, Yang-Laplace transform method Volterra and Abel's integro-differential equations of fractional order, Int. J. Nonlinear Anal. App., 9(2) (2018), 203-214, DOI: 10.22075/ijnaa.2018.13630.1709.

${ }^{[36]}$ F. Usta, A mesh free technique of numerical solution of newly defined conformable differential equations, Konuralp J. Math., 4(2) (2016), 149-157.

[37] M. Yavuz, T. A. Sulaiman, F. Usta, H. Bulut, [Analysis and numerical computations of the fractional regularized long wave equation with damping term, Math. Meth. Appl. Sci., In Press, DOI: 10.1002/mma.6343.

[38] G. Wu, D. Baleanu et al., Lattice fractional diffusion equation in terms of a Riesz-Caputo difference, Physics A., 438 (2015), 335-339.

[39] X. Zhang, L. Liu, Y. Wu, The uniqueness of positive solution for a fractional order model of turbulent flow in a porous medium, Appl. Math. Lett., 37 (2014), 26-33.

[40] A. R. Aftabizadeh, Y. K. Huang, N. H. Pavel, Nonlinear third-order differential equations with anti-periodic boundary conditions and some optimal control problems, J. Math. Anal. Appl., 192 (1995), 266-293.

[41] M. Yavuz, N. Özdemir, H.M. Baskonus, Solutions of partial differential equations using the fractional operator involving Mittag-Leffler kernel, Eur. Phys. J. Plus, 133(6) (2018), 1-11.

[42] M. Yavuz, Characterizations of two different fractional operators without singular kernel, Math. Model. Nat. Phenom, 14(3) (2019), 302.

[43] M. Yavuz, N. Özdemir Comparing the new fractional derivative operators involving exponential and Mittag-Leffler kernel, Discrete Contin. Dyn. Syst. Ser. S, 13(3) (2020), 995-1006.

[44] A. Yokus, Construction of different types of traveling wave solutions of the relativistic wave equation associated with the Schrödinger equation, Math. Model. Numer. Simul. Appl., 1(1) (2021), 24-31.

${ }^{[45]}$ P. Kumar, V.S. Erturk, Dynamics of cholera disease by using two recent fractional numerical methods, Math. Model. Numer. Simul. Appl., 1(2) (2021), 102-111. 